\title{
Specific Absorption Rate Measurement of External Heating Antennas Using Infrared Thermography
}

\author{
Chakaravarthi Geetha ${ }^{1}$, Arunachalam Kavitha ${ }^{1}$ \\ ${ }^{1}$ Department of Engineering Design, Indian Institute of Technology Madras, \\ Chennai, Tamil Nadu 600036, India \\ c.geetha1985@gmail.com, akavitha@iitm.ac.in
}

The role of thermal therapy as an effective adjuvant for radiation and/or chemotherapy of cancer has been demonstrated in several randomized clinical trials. During thermal therapy, power delivered to the target tissue in the form of acoustic or electromagnetic (EM) waves is released as heat. Selective elevation of tumour temperature resulting in heat induced cell kill, increased blood perfusion and enhanced drug intake. For device quality control and treatment planning, it is essential to characterize power deposition pattern of the heating device prior clinical use. Antenna specific absorption rate (SAR) can be measured with relative ease in clinical settings on muscle phantoms using infrared thermography (IR). Though this indirect measurement of antenna SAR has been well studied for interstitial type applicators, limited literature is available on the limitations of this approach and/or guidelines for characterizing external heating devices.

In this study, we measured the SAR patterns of two external microwave heating antennas on muscle phantoms. Controlled high power of 26 Watts was delivered for $30 \mathrm{~s}, 45 \mathrm{~s}, 60 \mathrm{~s}$ and $90 \mathrm{~s}$ pulse duration, $\Delta t$ to the antenna irradiating muscle phantom. Experiments were conducted at $434 \mathrm{MHz}$ for the cavity backed patch antenna, and at $915 \mathrm{MHz}$ for the spiral antenna. The rise in equilibrium temperature of the phantom after power delivery in the measurement plane was used to calculate antenna SAR. Measured SAR patterns at $5 \mathrm{~mm}$ and $20 \mathrm{~mm}$ depths obtained for varying pulse durations were compared with simulated SAR obtained using 3D EM simulations. Measurements indicated that SAR for pulse duration of $\Delta t=60 \mathrm{~s}$ agreed well with simulation for the cavity backed antenna, and $\Delta t=45 \mathrm{~s}$ yielded good agreement for the spiral antenna with minimal thermal artifacts. SAR full width at half maximum (FWHM) for cavity backed antenna at $5 \mathrm{~mm}$ and $20 \mathrm{~mm}$ depths were $54.68 \mathrm{~mm}$ $51.18 \mathrm{~mm}$ respectively in simulation, and $49.47 \mathrm{~mm}$ and $43.75 \mathrm{~mm}$ respectively in experiments. SAR FWHM for $915 \mathrm{MHz}$ spiral at $20 \mathrm{~mm}$ depth was $32.32 \mathrm{~mm}$ in simulation, and $38.66 \mathrm{~mm}$ in experiment. The pulse duration derived for these external heating applicators operating at two different industrial, scientific and medical (ISM) frequency is relatively higher compared to the duration used for interstitial thermal therapy applicators ( $\Delta t$ $\leq 30$ s). A theoretical study simulating antenna SAR measurements using thermographic technique is being carried out to assess if lesser pulse duration could yield accurate SAR patterns thereby, provide guidelines for characterizing external heating devices using IR thermography.

Keywords: specific absorption rate, thermal therapy, antennas, thermal artifacts, IR thermography. 